\title{
The reverse connectivity pattern between Broca's area and the left visual word form area in the processing of Chinese words and English characters
}

\author{
Yi-Yuan Tang*1,2, Shigang Feng1, Qingbao ${ }^{*}{ }^{1}$ and Qilin $\mathrm{Lu}^{1}$
}

\author{
Address: ${ }^{1}$ Institute of Neuroinformatics and Laboratory for Brain and Mind, Dalian University of Technology, Dalian 116024, China and \\ ${ }^{2}$ Department of Psychology, University of Oregon, Eugene, OR 97403, USA \\ Email: Yi-Yuan Tang* - yiyuan@uoregon.edu \\ * Corresponding author
}

from Seventeenth Annual Computational Neuroscience Meeting: CNS*2008

Portland, OR, USA. 19-24 July 2008

Published: II July 2008

BMC Neuroscience 2008, 9(Suppl I):P33 doi: I0.I I86/I47I-2202-9-SI-P33

This abstract is available from: http://www.biomedcentral.com/I47I-2202/9/SI/P33

(c) 2008 Tang et al; licensee BioMed Central Ltd.

\section{Background}

A recent notable controversy in language processing is whether there is a particular brain area specialized for visual word recognition within the visual ventral stream. Some researchers proposed that the left middle temporal fusiform gyrus is particularly responsible for visual word processing and then named this area as the Visual Word Form Area (VWFA) [1], while others disagreed [2]. Three types of stimuli in Chinese and English - Real characters (semantic and orthographic), Pseudo characters (orthographic), and Artificial characters (similar part feature) were examined in a reading task. Fourteen Subjects (seven females, seven males), all aged 19-22 years, with a university education, and fully right-handed, participated in the study. We recruited a $1.5 \mathrm{~T}$ fMRI GE scanner to acquire imaging data. Data analysis was performed with SPM99. After being realigned, normalized, smoothed $(6 * 6 * 8$ $\mathrm{mm})$, and low-pass filtered, a random-effect group analyses was conducted by using a one-sample t-test in the basic model toolbox. The within-condition interregional covariance analysis (WICA) method for Region of Interest (ROI)-based functional connectivity analysis was used to demonstrate the connectivity pattern between Broca's area and the left visual word form area in the processing of Chinese words and English characters $[3,4]$. We hypothesized that the left VWFA may orchestrate with Broca's area to respond to the different task demands.

\section{Results and conclusions}

The activation map was consistent with the previous findings with Chinese words and English characters processing while the connectivity analysis between Broca's area and the left VWFA indicated a reverse pattern. Our results provide strong evidence for VWFA in processing Chinese characters similar to that found in alphabetic scripts and hence support the hypothesis that left VWFA cooperates with Broca's area to respond to the different task demands [5].

\section{Acknowledgements}

This work was supported in part by National Natural Science Foundation of China Grant 30670699, Ministry of Education Grant NCET-06-0277 and 021010.

\section{References}

I. Cohen L, Lehericy S, Chochon F, Lemer C, Rivaud S, Dehaene S: Language-specific tuning of visual cortex functional properties of the visual word form area. Brain 2002, 125:1054-1069.

2. Price CJ, Devlin JT: The myth of the visual word form area. Neuroimage 2003, 19:473-48I.

3. He GJ, Tan LH, Tang YY, James GA, Wright P, Eckert M, Fox PT, Liu YY: Modulation of neural connectivity during tongue movement and reading. Human Brain Mapping 2003, 1 8:222-232.

4. Tang YY, Zhang WT, Chen KW, Feng SG, Ji Y, Shen JX, Reiman RE, Liu YY: Arithmetic processing in the brain shaped by cultures. Proceedings of the National Academy of Sciences of the USA 2006, 103: $10775-10780$.

5. Liu C, Zhang WT, Tang YY, Mai XQ, Chen HC, Tardif T, Luo YJ: The visual word form area evidence from an fMRI study of implicit processing of Chinese characters. Neurolmage 2008 in press. 